\title{
On the Communication Requirements for the Smart Grid
}

\author{
Mohamed Daoud, Xavier Fernando \\ Electrical and Computer Engineering Department Ryerson University, Toronto, Canada \\ E-mail: Mohamed.Daoud@Ryerson.ca \\ Received November 5, 2010; revised December 15, 2010; accepted January 7, 2011
}

\begin{abstract}
The current power grid is facing many challenges that it was not designed or engineered to handle which range from congestions and major blackouts to the overwhelming increase in demand and security concerns. The current electric grid was established before the 1960's. It is believed that the electric grid is the most complex and gigantic machine ever made in human history; it consists of wires, cables, towers, transformers and circuit breakers installed together in outdated manner. During the 60's, computers and sensors were used to monitor and slightly control the grid; however, fifty years later these sensors are considered less than ideal. Presented here is a review of the smart grid communication network in terms of configuration, bandwidth and latency requirements as well as the technology used. We simulate the access layer of the smart grid network and show that no single available communication technology can be used for all layers of the smart grid; thus, different technologies for different layers are needed. A new protocol for optimizing the smart grid is recommended.
\end{abstract}

Keywords: Smart Grid, Core Network, Distribution Network, Access Network, Energy, Latency, Bandwidth

\section{Introduction}

Climate change and global warming have been among the major concerns of human beings over the last few years. Recently huge efforts have gone into integrating renewable and green sources of energy with the grid and making the grid smarter; that is, to increase its energy efficiency, storage and moderate energy consumption.

To that extent reducing and shifting peak load are essential to support renewable energy generation and would lead to shutting down the extra, carbon-intensive power plants that are used only during peak hours. Therefore, governments along with utilities have put into effect mechanisms to reduce peak demand, including time-of-use pricing, installation of load management devices, load shifting and peak eliminating technologies.

The Smart grid designed for the future electricity system encompasses many of these solutions and technologies. It empowers energy consumers as well as utilities to gain better control over energy consumption.

However, it seems that these are all impossible without considering the key role of communication technology. In fact, various communication technologies have the potential to revolutionize today's grid and expedite renewable energy projects.
If we look at the electric grid in the United States we will see that it consists of 3,100 electric utilities operating more about 10,000 power plants and 131 million customers consuming more than 3,500 billion kwh every day [1,2]. Between them there are 157,000 miles of high voltage electric transmission lines [1]. The average age of the power grid transmission lines is 50-60 years [3]. In the decade from 1988 to 1998, the electricity demand in the U.S grew by $30 \%$, yet only $15 \%$ of new transmission capacity was added [4]. This is a giant machine that needs to be operated efficiently to save both resources and the environment. But due to its age, being somehow deregulated and inefficient it is very hard to solve this optimization problem.

The current system is inefficient because when a fault occurs the utility does not know unless the customers call in. Also because of the congestion and many blackouts that take place regularly, such as the one in the summer of 2003 in the Northeastern US and in Canada. Power outages and power quality issues cost U.S businesses more than $\$ 100$ billion on average each year [3]. Power inefficiency has a negative impact on the environment as well. To be more specific, roughly 40 percent of America's total $\mathrm{CO}_{2}$ emissions come from the production of electricity used in homes, offices, and factories [5]. To 
add intelligence to an electrical power transmission system, we need to have independent processors in each component and at each substation and power plant. These processors must have a robust operating system and be able to act as agents that can communicate and cooperate with each other to compose a large distributed computing platform [6].

No one clear definition for the Smart Grid can be found, but it can be described as Advanced Metering Infrastructure (AMI) accompanied by substation and distribution automation services and enhanced distribution and outage management. This shows the wide range of requirements and expectations from the Smart Grid. The Electric Power Research Institute (EPRI) defines the Smart Grid as, “A power system that serves millions of customers and has an intelligent communications infrastructure enabling timely, secure and adaptable information flow needed to provide power to an evolving digital economy" [7]. Smart Grid will offer a system-wide "macro" view in aid of conserving electrical energy within the grid and related distribution systems [8], by controlling some home appliances such as the thermostat of the air conditioners, charging the Plug in Hybrid Electrical Vehicle (PHEV), and switching the washing machines, dryers, and dishwashers to low demand times when the hydro is less expensive. These appliances will be smart and equipped with a special chip; the smart appliances could talk to the grid and decide how to operate best and automatically schedule their activities at strategic times based on available generation [9]. The smart meter will play an important role here; also the existence of a robust reliable communication network is essential.

Smart Grid can be described as an energy network, a network just like the internet. Rather than downloading and uploading data, customers will download and upload electricity [10]. Rather than having a modem indicating how many megabytes of data downloaded or uploaded, customers will have smart meters showing the kilowatts they used or generated and the price according to the time of use. Smart Grid is information technology infrastructure meeting electrical infrastructure to satisfy future energy needs; it will combine the maturity of the electric grid with the efficiency, connectivity, and cost gains brought about by information technology [11]. IEEE recently took the initiative to define the standards and guidelines for the Smart Grid; IEEE P2030 was formed for that purpose [2].

According to the United States Department of Energy's Modern Grid Initiative report, a modern smart grid must:

1) Be able to heal itself

2) Motivate consumers to actively participate in operations of the grid
3) Resist attack

4) Provide higher quality power that will save money wasted from outages

5) Accommodate all generation and storage options

6) Enable electricity markets to flourish

7) Run more efficiently

8) Enable higher penetration of intermittent power generation sources

This paper presents a complete review of the communications requirements for the smart grid and the work done so far.

The rest of the paper is organized as follows: Section 2 presents the hierarchy of the communications system and also discusses latency, bandwidth, and Quality of Service (QoS). Section 3 is a description for all three layers of the network. Section 4 presents some of the work already done. Finally Section 5 concludes our review and gives a glimpse of some future work.

\section{Communications for Smart Grid}

Integrated, high performance, highly reliable, scalable, ubiquitous, and secure-these are the characteristics describing the smart grid communication network. The communication network will be responsible for gathering and routing data, monitoring all nodes and acting upon the data received.

Apparently the amount of data transferred daily will be huge and very sensitive, so the communication network must be secured against external attacks. Smart grid communication network will depend on both wireless and wired communication technologies; however, one of the main challenges of the smart grid communication network is that wireless technologies are totally turning over every 4-6 years and utilities are building systems for 15-20 years. This is why unifying and converging networks around IP is so critical. Hence arises the idea of communication networks not being tied to a specific carrier technology. Another challenge is the security; keeping all this information secured and preventing hackers from getting into the grid is truly a matter of national security.

In [10], S. Keshav and C. Rosenberg compared the smart grid communication network to the internet, which is the largest and most important communication network on earth. One of the main differences is the availability; the internet is not available at every house, but the smart grid should be available to every house with high reliability. The smart grid communication network will be like the internet in the sense of being a delay tolerant network, providing congestion control, and operating in distributed control manner. 


\subsection{The Communications Network Hierarchy}

Smart Grid communication network will be a network of networks that may use different communications technology or just one, allowing two-way, reliable, and secure communications.

It will be formed of millions of smart meters at customer premises connected to a few thousand substations, which in turn will be connected to fewer control centers and power plants. The network will be huge; thus, it is recommended to take the form of clusters according to geographical locations. Each cluster will have a limited number of smart meters ranging from a few hundred to a few thousand connected to a few substations and control centers. A cluster may or may not have a power plant as power plants will be shared between more than one cluster.

There will be three different layers in the communications network, as described in [12].

Core network handles connectivity between substations and utilities' head offices i.e. control centers.

Distribution network as shown in Figure 1, handles broadband connectivity for transmitting data collected by the smart meters sensors and concentrators located on the grid to their related databases and analytics servers, which are located at headquarters.

Access network handles last-mile connectivity at homes, offices, and municipal facilities to the smart meters.

Millions of messages every second will be going back and forth in the network; different messages for different reasons can be sorted into three main categories $[13,14]$.

- Real-Time Operational communication requirements

- Administrative Operational communication requirements

- Administrative communication requirements

Real-Time Operational communication is the communication in real time required to maintain operation of the power system [13]; it is the control and protection for

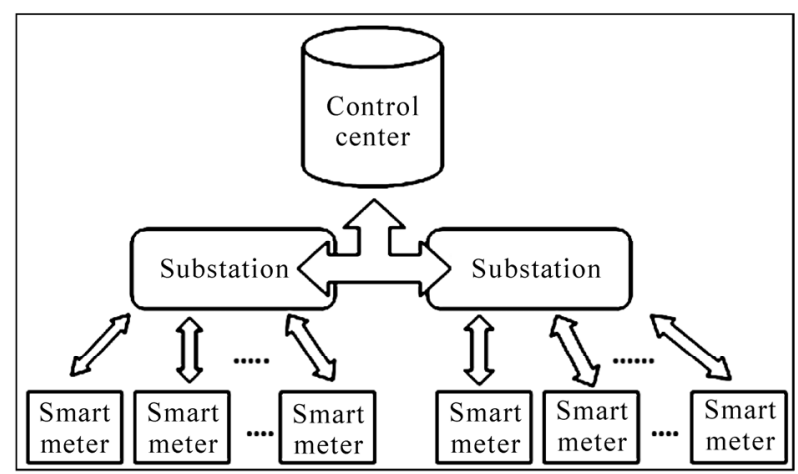

Figure 1. Smart grid distribution network. messages. It requires low latency and has a highly successful delivery rate.

Administrative Operational communications requirements are usually those messages that describe major and minor system disturbances like local event recorders, disturbance recorders, and power swing recorders. These do not need to take place in real time. This type of information is needed to predict future demand.

Administrative communication requirements include the voice communications between different locations for administrative purposes. This type of communication can be carried over cellular or land line networks; it does not necessarily need to be part of the smart grid communications network.

These different categories point out one of the main requirements for the smart grid communication network which is supporting QoS in order to prioritize traffic on the network.

\subsection{Requirements for Smart Grid Communication Network}

Latency is the delay in the network or the expression of how much time it takes for a packet of data to travel from one point on the network to another. There is a need for a communication infrastructure with exceptionally tight latency characteristics as it is one of the most stringent requirements for the grid. If the control center misses any input, then it might substitute the missing input with inputs from other sensors which can produce different actions that could lead to erroneous results [15]. K. Moslehi et al. [16] discussed latency within the smart grid communications network. They explained that the network will have different latency times; the grid is huge so if the data sent is for the purpose of system wide coordinated controls it should have higher latency (slower cycle) than if the data is required for local analytical needs or responding to rapid events (faster cycle).

Bandwidth: it is extremely important to determine the bandwidth requirements for the smart grid communication network because it is a direct factor when choosing the transmission media (e.g. fiber optics, radio waves, coaxial cables, etc.) as well as choosing the communications technology (e.g. 3G, LTE, WiMAX etc.). IEEE P2030 standard is still trying to define the bandwidth requirements [17]. An important point here is that because of the extremely large number of endpoints, the communications system bandwidth requirements can quickly become untenable if appropriate precautions are not taken.

QoS: Not all messages have the same importance nor should they be delivered within the specific latent period.”) 


\section{Layers of the Network}

\subsection{Core Network}

In $[18,19]$, K. Moslehi et al. gave an example of a Distributed Autonomous Real Time (DART) smart grid network consisting of 10 regions, each region having 20 control centers; each control center is connected to 500 substations, according to the geographical areas. Each 10 substations can be grouped into a zone.

According to the DART system in $[18,19]$, the latency calculated in the control area between the 500 substations and their control center is 240.8 msec. This value measures latency in the system but does not indicate the maximum tolerable latency.

In [15], V. k. Sood et al. discussed the latency within smart grids and concluded that faults require continuous high rate monitoring by the control centers. For rapid detection of such faults the latency should be in the order of tens of milliseconds, with $100 \mathrm{msec}$ being the accepted fault detection time in medium sized systems.

For bandwidth calculation purposes, a snapshot taken at the control area in the DART system $[18,19]$ shows that the required transfer rate is $5.089 \mathrm{MB} / \mathrm{sec}$. This number may seem large due to the extensive DART system where 500 substations are connected to one control center, but a smaller system as in [15] with three voltages and three currents to be sampled and sent to a control centre have only a 2-5 Mbits/s bandwidth requirement. This data rate is indicative of an application with a relatively low to medium data rate production.

Fiber optics can be used for communication in the core network. They were recommended in [15] because of their very low latency of under 5 usec latency per kilometer length of strand. But fiber is not available to all grid operators, and not all points in the system can have fiber cables extended to them. In [20] the author agrees with the above argument and suggests optical fiber because of its low latency; however, the problem again seems to be deploying the fiber optics all over the network and to the customer premises. The author highlighted the idea that different technologies can be used for different parts of the network as long as they can talk to each other i.e. based on IP. Since the core network is the part that handles connectivity between substations and utilities' head offices/ control centers, so installing fiber optics will not be as difficult because the number of substations and head offices is relatively small and usually built in specific locations carefully chosen by the utility company.

\subsection{Distribution Network}

The following calculations done in $[18,19]$ for the DART system measure the latency within the substation: between smart meters and a specific substation is $2.2 \mathrm{msec}$ and in the zone between substations is $4.8 \mathrm{msec}$, but this is not the maximum tolerable latency.

The maximum tolerable latency is higher; the latency is in the order of a few milliseconds, around $10 \mathrm{msec}$ [2] while in [18] it was assumed to be $12 \mathrm{msec}$; i.e. $6 \mathrm{msec}$ one-way delay. These latency requirements change significantly in case of islanding. Islanding is the condition where the power grid is broken into independent asynchronous sections, each having its own generators and loads. According to the IEEE standard 1547-2003 the Distributed Resource (DR) must detect the unintentional islands and cease to energize them within 2 seconds of the formation of the island [15]. Unintentional islanding may lead to abnormal voltage and frequency change out of the acceptable range. In [15] the latency in case of islanding was estimated to be maximum 6 cycles or $100 \mathrm{msec}$.

Pramode Verma et al. [2] proposed a method to calculate the bandwidth in distribution network by assuming a system of one transmission substation connected to one distribution substation and control center connected to 10,000 feeders. Each feeder is connected to 10 smart meters, making a total of 100,000 smart meters each sending one message per second in addition to the control messages. Thus, in the case of busy hour the system may have one million messages per second. Assuming each message is 100 bits, the latency requirement is $10 \mathrm{msec}$ and the messages follow a Poisson discipline at each node, bandwidth is calculated to be $100.01 \mathrm{Mbps}$. Changing the delay requirement to $10 \mathrm{msec}$ for $99 \%$ of the messages causes the bandwidth to increase to $100.056 \mathrm{Mbps}$. Repeating the same calculations for a 400 bit message, the bandwidth was found to be $400.04 \mathrm{Mbps}$, but if the delay is limited to $10 \mathrm{msec}$ for $99 \%$ of the messages the bandwidth increases to $400.086 \mathrm{Mbps}$. It was concluded that both of these situations result in very poor bandwidth utilization, while a higher level of utilization will not meet the assumed latency constraint [2].

Utilizing the bandwidth is an important issue that needs to be carefully studied; Carl H. Hauser et al. [21] proved that a $\mathrm{T} 1$ line carrying a 400 bit message with latency constraint of $10 \mathrm{msec}$ results in utilizing $6 \%$ only of the $\mathrm{T} 1$ line.

In $[16,18]$, the required transfer rate was found to be 3.31 MB/sec in the substation and $8.1 \mathrm{MB} / \mathrm{sec}$ within a zone formed of 10 geographically grouped substations In Distributed Autonomous Real Time (DART) system which they proposed, the maximum data transfer rate required is $8.1 \mathrm{MB} / \mathrm{sec}$. According to the analysis, the size of data for a snapshot describing the instantaneous status can vary between 2.5 kBytes for a substation to 250 Mbytes for the entire grid [18]. This type of infor- 
mation is very useful for determining the bandwidth.

As a communication technology for the distribution network, WiMAX seems to be a very good candidate as it has the benefits of fiber, such as low latency and large bandwidth. At the same time it can be easily deployed as it needs no line of sight and no expensive physical infrastructure as fibers do. Other benefits of WiMAX include the following: it provides higher speed than $3 \mathrm{G}$, it is an emerging broadband wireless access technology and it can provide high-speed connection to internet, with data transmission less than $50 \mathrm{~km}$ [22]. One of the most important advantages of WiMAX, besides the high transmission rate is the QoS guarantees, also the adaptive modulation and closed loop power control are very attractive options. In the Smart Grid some messages may be more important than others - the control messages should have higher priority than billing messages for example [22]. WiMAX is an attractive solution to be used in the communication network since WiMAX provides longer distance communications (10-30 miles) with a data transfer rate of 75 Mbps while communicating out of sight. This system also communicates point to point with different vendors, and the authors added that it may be used as the spine of transmission and distribution communications system [23]. In [15], the authors expressed their interest in wireless technologies, especially 4G like WiMAX and LTE as both can provide low latency and high bandwidth. Moreover the QoS, ensures that traffic can be prioritized on the network also they are built on IP. In [15], it was mentioned that wireless technology 4G especially WiMAX can be used for transferring data from smart meters from homes to transformer stations and control centers as it will give high speed and low latency. Latency in a WiMAX link from the base station to CPE (customer premises equipment) is typically equal to or less than $10 \mathrm{~ms}$ [15].

WiMAX will easily satisfy the bandwidth requirements of the distribution network as it offers large bandwidth ranges between $5 \mathrm{MHz}$ to $20 \mathrm{MHz}$.

\subsection{Access Network}

The smart meter will not only show the customer's usage and generation, but will also collect information from the smart appliances at home through an access network indicating the customer's behavior and informing the grid of any increase or decrease in demand.

The amount of data will depend on the number of smart appliances in the home; the more smart appliances, the more bandwidth needed. Figure 2 shows a smart meter collecting data from smart appliances through the access network and sending this data to the substation through the distribution network. Not all appliances will

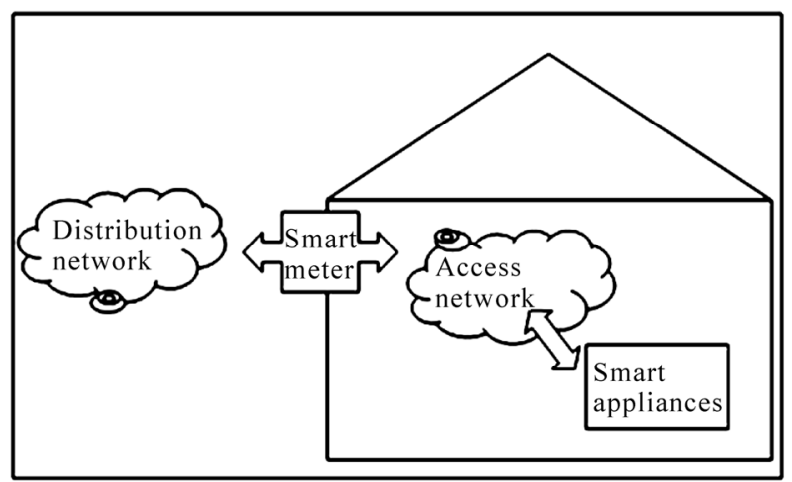

Figure 2. Smart meter collecting data from the access network inside a house and passing it to the distribution network.

be sending/receiving data at the same time. They will send/receive at scheduled times or when needed. It will be easy to design and manage the access network because of its small size and the limited amount of data to be transferred.

In [20] an in home network was mentioned where the smart appliances can communicate to the smart meter with a data rate of $20 \mathrm{Kbps}$ while the maximum data rate is $128 \mathrm{Kbps}$.

Such network can use any short range communication technology like ZigBee or bluetooth. In fact ZigBee is preferred because it is an open standard protocol based on IEEE802.15.4 which is a high level communication protocol using small low power digital devices designed for low cost and low power communications. Because ZigBee can activate (go from sleep to active mode) in 15 msec or less, the latency can be very low. Because ZigBees can sleep most of the time, average power consumption can be very low, resulting in long battery life. Zigbees have a small range and limited bandwidth and the data rate isn't very high compared to fiber as an example, so they are more suitable for indoor applications like home automation. This technology is even preferred over Bluetooth in the short range applications because it consumes far less power. The obvious result is an increase in the life expectancy of the network [24].

Zigbee radio nodes are self organizing and self healing when forming mesh networks. Given the fact that IEEE 802.15.4 radios can successfully transmit packets a distance of 50 meters - nearly half the length of a football field-the meters can form either a mesh or star network with other meters in the neighborhood [24]; 50 meters is sufficient to allow communication between smart appliances and the smart meter at home. Zigbee is both power and cost efficient.

We did a simulation for an access network where we assumed a typical mid-house size with 11 smart devices communicating through a Zigbee network to the smart 
meter. We assumed Poisson distribution for packets generation at the smart devices with a constant packet size of 1 Kbyte. Packets are sent to the smart meter as soon as they are generated at the smart devices. We simulated 24 hours of traffic on the access network and found that the end-to-end delay in the network ranged between $35 \mathrm{msec}$ and $80 \mathrm{msec}$, with a spike of $0.1 \mathrm{sec}$ during the peak hour of the day. Our results are shown in Figure 3. We calculated the average Bit Error Rate (BER) at the smart meter and we got $\mathrm{BER}=0.06=6 \%$. In Figure 4 we plotted the data throughput over the day; it ranged from $90 \mathrm{Kbps}$ to $100 \mathrm{Kbps}$ which shows that the minimum required bandwidth should be a little bit over $100 \mathrm{Kbps}$.

A Smart grid communication network will consist of different layers each using a different technology; thus, all these technologies should be able to communicate together using the same protocol. For best performance,



Figure 3. Average end-to-end delay in the access network.

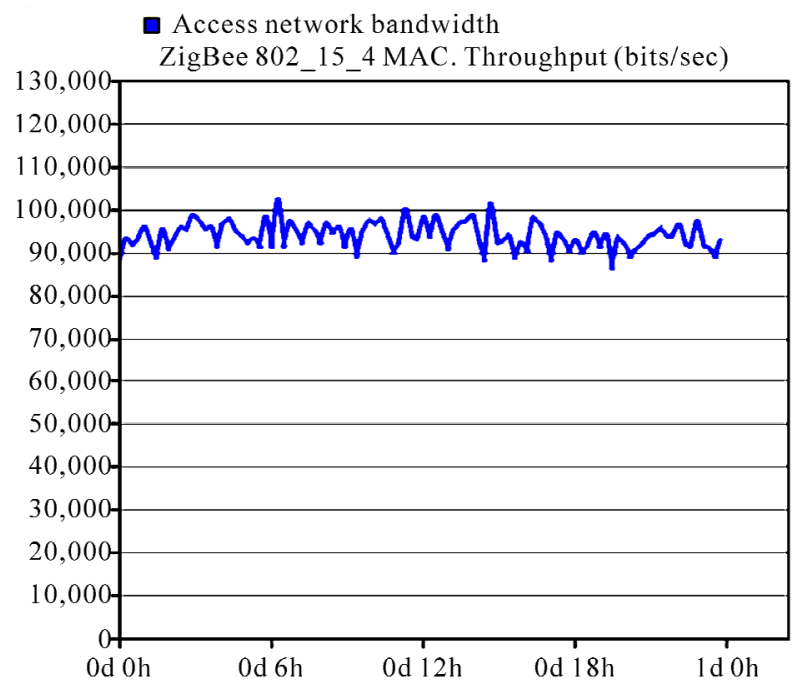

Figure 4. Bandwidth of the access network. all layers should be IP networks.

IP networks are widely used because of their open standard, simplicity, reliability, security, and robustness. The world is going to the all-IP networks concept. IP is being used in internet, computer networks, cellular networks, Wi-Fi, 3G, LTE (Long Term Evolution) and almost all new technologies where it provides a low cost and efficient solution. On the other hand, there is Asynchronous Transfer Mode (ATM), which is a packetswitching technology that delivers data packets over virtual circuits or preserved paths through the network [25]. ATM is more expensive than IP, but it provides guaranteed latency and drop rates. ATM is used as the backbone of IP networks to implement point to point links.

IP seems to be a more attractive solution for smart grid networks than ATM in terms of the ability to interact with other communication networks and the internet. It is also in an economical method, as the cost of deployment and maintenance can be reduced significantly with the use of IP-based technologies [2,20].

When talking about IP it is necessary to mention the layers on top of it. IP is usually backed with Transmission Control Protocol (TCP) to provide a higher delivery rate and retransmission in case of lost data [15,21,22,25]. TCP is well known for having the highest level of packet delivery assurance, but this comes at a price of higher latency duty to the large overhead $[15,21]$. But it can still be used in combination with prioritization through QoS and used for highly important applications that need assured delivery. User Datagram Protocol (UDP) is another layer that comes on top of IP, but in contrast to TCP, UDP is lightweight with smaller overhead and latency than TCP but at the cost of non assured data delivery because receipts are not acknowledged. A practical application of UDP is the multimedia features over the internet, where the loss of some packets can be tolerated. By analogy the same concept can be used for smart grid communication network; TCP could be used for messages that require high delivery rate like control messages while UDP can be used for sending data where the loss of some packets will not affect the overall performance of the system.

Currently most IP network are based on IPv4, but IPv6 protocol can be used; it has an address code set at 128 bit, which means that there are $2^{128}$ IP addresses available. IPv6 is supposed to be faster than internet IPv4, and it will maintain dialogue with any object such as household appliances, sensors and so on [22]. Although the IPv4 extensions allow multicast traffic and certain QoS, IPv6 is still preferred as it includes the following services and new features: more addresses, mobility, security, etc [20]. It is recommended having the Smart Grid communication network as a separate entity from the internet as in $[2,21]$ where it was concluded that the public internet 
will lack admission control and guaranteed latency delivery and will never be able to supply private data network for the power grid infrastructure needs. The lack of security of the internet is another major concern.

\section{Current Projects}

Many research projects and activities have been done in the area of smart grids, and some of them are listed in this section.

GAD project in Spain is targeting residential consumption. They developed a Domestic Power Manager (DPM) which is much like the smart meter, and took many steps in defining a communication network using open standard protocols to support active demand side management $[20,24]$.

GridStat is being developed by Washington State University. They are offering a flexible approach to providing communication support for electric power grid operations. It is based on a publish-subscribe (pub-sub) model, where the substations periodically publish status while the control centers and other substations subscribe to a selected set of statuses [21,25].

DisPower, CRISP, MicroGrid and Fenix are different projects adopting the concept of an internet-like network in the sense that decision making is distributed all over the network since the control nodes are spread across the system [26].

Modern grid strategy [27] is a project by the U.S department of Energy (DOE) that started in 2005 through the National Energy Technology Laboratory (NETL). They are developing smart grid concepts and sharing it with key stock holders. Their mission is to accelerate grid modernization in the United States. They support the idea of using different communication technologies in different layers of the smart grid.

IntelliGrid is an initiative by EPRI to create the technical foundation for a smart power grid that links electricity with communications and computer control to achieve tremendous gains in reliability, capacity, and customer services. A major early product is the IntelliGrid Architecture, an open-standard, requirements-based approach for integrating data networks and equipment that enables interoperability between products and systems. This program provides utilities with the methodology, tools, and recommendations for standards and technologies when implementing systems such as advanced metering, distribution automation, demand response, and wide-area measurement [28].

\section{Conclusion}

This paper presented a review of communications for smart grids in which the specific needs of the smart grid communication system were discussed. The hierarchy of the system, nature of the network, latency, bandwidth and proposed communication technologies to be used were all surveyed. Current active projects were mentioned as well. It was found that the latency within the distribution network should be kept at $10 \mathrm{msec}$, and the required transfer rate in case of a zone containing 10 substations should be $8.1 \mathrm{MB} / \mathrm{sec}$. Our simulation results for the access layer in a smart grid network point out the access layer requirements in terms of end-to-end delay, and bandwidth. Also it was concluded that no single communication technology will be able to satisfy the requirements for the whole network; rather different technologies should be used for different parts.

There is still much work to be done in the smart grid area, especially in the communications part. Since all of the available communication techniques are off the shelf technologies designed for different reasons, none of them addresses the smart grid needs. Most of these technologies support mobility, handover, and many other features which are not needed for the smart grid due to its nature; thus, a communication protocol should be developed and optimized specially for the smart grids that cover end-toend networks. This special protocol should be able to automatically set the QoS configurations when application requirements change based on the grid events, and it should translate the self-healing grid capability to selfhealing communication network.

\section{References}

[1] L. H. Tsoukalas and R. Gao, "From Smart Grids to an Energy Internet: Assumptions, Architectures and Requirements," Third International Conference on Electric Utility Deregulation and Restructuring and Power Technologies (DRPT 2008), Nanjuing, 6-9 April 2008, pp. 94-98. doi:10.1109/DRPT.2008.4523385

[2] A. Aggarwal, S. Kunta and P. K. Verma, "A Proposed Communications Infrastructure for the Smart Grid,” Innovative Smart Grid Technologies (ISGT), Gaithersburg, 19-21 January 2010, pp.1-5.

[3] V. C. Gungor, B. Lu and G. P. Hancke, “Opportunities and Challenges of Wireless Sensor Networks in Smart Grid - A Case Study of Link Quality Assessments in Power Distribution Systems," IEEE Transactions on Industrial Electronics, Vol. 57, No. 10, October 2010, pp. 3557-3564. doi:10.1109/TIE.2009.2039455

[4] C. W. Gellings, "The Smart Grid: Enabling Energy Efficiency and Demand Response,” Fairmont Press, Lilburn, 2009.

[5] T. L. Friedman, "Hot, Flat, and Crowded: Why we need a green revolution and how it can renew America,” Farrar Straus \& Giroux, New York, 2010. 
[6] S. M. Amin and B. F. Wollenberg, "Toward a Smart Grid: Power Delivery for the 21st Century," IEEE on Power and Energy Magazine, Vol. 3, No. 5, September-October 2005, pp. 34-41. doi:10.1109/MPAE.2005.1507024

[7] D. Mayne, "How the Smart Grid Will Energize the World,” White Paper. http://www.digi.com

[8] B. Rob and B. Tim, "Practical Consideration of the Smart Grid,” White Paper. http://www.wireie.com

[9] M. A. S. Masoum, P. S. Moses and S. Deilami, "Load Management in Smart Grids Considering Harmonic Distortion and Transformer Derating," Innovative Smart Grid Technologies (ISGT), Gaithersburg, 19-21 January 2010, pp.1-7.

[10] S. Keshav and C. Rosenberg, "How Internet Concepts and Technologies can Help Green and Smarten the Electric Grid," Proceedings of the 1st ACM SIGCOMM Workshop on Green Networking, 2010, pp. 35-40. doi:10.1145/ 1851290.1851298

[11] P. Venkat and M. Saadat, "Smart Grid: Leveraging Intelligent Communications to Transform the Power Infrastructure," White Paper.

[12] Alvarion Research and Development Team-Smart Grid Unit, "Optimizing Smart Powergrids with WiMAX and Broadband Wireless Connectivity Solutions," White Paper. http: //www.alvarion.com

[13] G. N. Ericsson, "Cyber Security and Power System Communication - Essential Parts of a Smart Grid Infrastructure," IEEE Transactions on Power Delivery, Vol. 25, No. 3, July 2010, pp. 1501-1507. doi:10.1109/TPWR D.2010.2046654

[14] G. N. Ericsson, "Classification of Power Systems Communications Needs and Requirements: Experiences from Case Studies at Swedish National Grid,” IEEE Transactions on Power Delivery, Vol. 17, No. 2, April 2002, pp. 345-347. doi:10.1109/61.997896

[15] V. K. Sood, D. Fischer, J. M. Eklund and T. Brown, "Developing a Communication Infrastructure for the Smart Grid,” IEEE on Electrical Power \& Energy Conference (EPEC), Montreal, 22-23 October 2009, pp.1-7. doi:10. 1109/EPEC.2009.5420809

[16] K. Moslehi, A. B. R. Kumar, D. Shurtleff, M. Laufenberg, A. Bose and P. Hirsch, "Framework for a Self-Healing Power Grid," IEEE on Power Engineering Society General Meeting, Vol. 3, 12-16 June 2005, p. 3027. doi:10. 1109/PES.2005.1489709

[17] M. E. Ropp, "Similarities between Vehicle-to-Grid Interfaces and Photovoltaic Systems," IEEE on Vehicle Power and Propulsion Conference (VPPC'09), 7-10 September 2009, pp. 1221-1225. doi:10.1109/VPPC.2009.5289711

[18] K. Moslehi and R. Kumar, "Smart Grid — a reliability perspective,” Innovative Smart Grid Technologies (ISGT),
Gaithersburg, 19-21 January 2010, pp.1-8. doi:10.1109/ ISGT.2010.5434765

[19] K. Moslehi, "Transmission Fast Simulation and Modeling: "Functional Requirements,” 1011666, “Architectural Requirements”, 1011667, and "Business Case Analysis”, 1012152, EPRI, Palo Alto, CA, Intelligrid Final Report, October 2005. http://www.epri-intelligrid.com

[20] F. Lobo, A. Cabello, A. Lopez, D. Mora and R. Mora, "Distribution Network as Communication System," SmartGrids for Distribution, IET-CIRED, CIRED Seminar, 2324 June 2008, pp. 1-4.

[21] C. H. Hauser, D. E. Bakken, I. Diony-Siou, K. H. Gjermundrod, V. S. Irava, J. Helkey and A. Bose, ”Security, Trust, and QoS in Next-Generation Control and Communication for Large Power Systems,” International Journal of Critical Infrastructures, Vol. 4, No. 1-2, 2008, pp. 3-16.

[22] Z. C. Xu and X. M. Li, "The Construction of Interconnected Communication System among Smart Grid and a variety of Networks," Power and Energy Engineering Conference (APPEEC), 2010 Asia-Pacific, 28-31 March 2010, pp. 1-5. doi:10.1109/APPEEC.2010.5448246

[23] S. M. Kaplan, F. Sissine, A. Abel, J. Wellinghoff, S. G. Kelly and J. J. Hoecker, "Smart Grid: Modernizing Electric Power Transmission and Distribution; Energy Independence, Storage and Security; Energy Independence and Security Act of 2007 (EISA); Improving Electrical Grid Efficiency, Communication, Reliability, and Resiliency; Integrating New and Renewable Energy Sources,” Part of the Government Series, US Federal Government Report, Capitol.Net, 2009.

[24] F. Lobo, A. Lopez, A. Cabello, D. Mora, R. Mora, F. Carmona, J. Moreno, D. Roman, A. Sendin and I. Berganza, "How to Design a Communication Network over Distribution Networks," 20th International Conference and Exhibition on Electricity Distribution - Part 1, 8-11 June 2009, pp. 1-4.

[25] C. H. Hauser, D. E. Bakken and A. Bose, “A Failure to Communicate: Next Generation Communication Requirements, Technologies, and Architecture for the Electric Power Grid,” IEEE on Power and Energy Magazine, Vol. 3, No. 2, March-April 2005, pp. 47-55. doi:10.1109/ MPAE.2005.1405870

[26] T. J. Hammons, "Integrating Renewable Energy Sources into European Grids,” International Journal of Electrical Power \& Energy Systems, Vol. 30, No. 8, October 2008, pp. 462-475. doi:10.1016/j.ijepes.2008.04.010

[27] U.S Department of Energy, National Energy Technology Laboratory (NETL). http://www.netl.doe.gov/smartgrid

[28] IntelliGrid Project, Electric Power Research Institute (EPRI). http://intelligrid.epri.com 